\title{
Pore Structure Characteristics and Evolution Law of Different- Rank Coal Samples
}

\author{
Zhihui Wen $\mathbb{D}^{1,2,3}$ Qi Wang $\mathbb{D}^{1,2}$ Yunpeng Yang, ${ }^{1,2}$ and Leilei Si ${ }^{1,2,3}$ \\ ${ }^{1}$ School of Safety Science and Engineering, Henan Polytechnic University, Jiaozuo 454000, China \\ ${ }^{2}$ State Key Laboratory Cultivation Base for Gas Geology and Gas Control (Henan Polytechnic University), Jiaozuo 454000, China \\ ${ }^{3}$ Collaborative Innovation Center of Coal Work Safety and Clean High Efficiency Utilization, Jiaozuo 454000, China
}

Correspondence should be addressed to Zhihui Wen; wenzhihui@hpu.edu.cn

Received 30 April 2021; Accepted 1 June 2021; Published 17 June 2021

Academic Editor: Feng Du

Copyright (C) 2021 Zhihui Wen et al. This is an open access article distributed under the Creative Commons Attribution License, which permits unrestricted use, distribution, and reproduction in any medium, provided the original work is properly cited.

In this study, the full-size pore structure characteristics of six different-rank coal samples were investigated and analyzed from three perspectives, namely, pore shape, pore volume, and pore specific surface area, by performing a high-pressure mercury injection experiment and a low-temperature nitrogen adsorption experiment. Next, the full-size pore volumes and pore specific surface areas of the six coal samples were accurately characterized through a combination of the two experiments. Furthermore, the relationships between volatile matter content and pore volume and between volatile matter content and pore specific surface area were fitted and analyzed. Finally, the influences of metamorphic degree on pore structure were discussed. The following conclusions were obtained. The pore shapes of different-rank coal samples differ significantly. With the increase of metamorphic degree, the full-size pore volume and pore specific surface area both decrease first and then increase. Among the pores with various sizes, micropores are the largest contributor to the full-size pore volume and pore specific surface area. The fitting curves between volatile matter content and pore volume and between volatile matter content and pore specific surface area can well reflect the influence and control of metamorphic degree on pore volume and pore specific surface area, respectively. With the increase of volatile matter content, the pore volume and the pore specific surface area both vary in a trend resembling a reverse parabola.

\section{Introduction}

China is a country boasting abundant coal resources. According to National Energy Development Strategy (20302050) formulated by the Chinese Academy of Engineering, coal resources will account for about 55\% of the whole energy structure of China in 2030 and will still account for over 50\% in 2050 [1,2]. That is, coal will still occupy the most important position in China's primary energy structure in the future [3]. Coal is a typical heterogeneous porous medium whose pore structure is one of the key parameters for evaluating it [4]. Pore structure not only determines the physical and mechanical properties of coal, but also controls the process of gas adsorption, desorption, diffusion, and seepage in a coal seam [5-7]. In particular, the adsorption capacity of coalbed methane (CBM) is closely related to the pore development degree and pore structure characteristics. Therefore, the research on the pore structure characteristics of coal is meaningful for gas disaster prevention and CBM extraction and utilization [8-10]. As specified by the Hodot decimal classification standard [11-13], the pores in coal can be divided into five categories according to the pore size, i.e., micropores $(<10 \mathrm{~nm})$, small pores $(10-100 \mathrm{~nm})$, mesopores $(100-1,000 \mathrm{~nm})$, macropores $(>10,000 \mathrm{~nm})$, and visible pores $(>100,000 \mathrm{~nm})$. Among them, micropores, also referred to as adsorption pores, are the main space for gas adsorption. The commonly used methods to measure the pore structure characteristics of coal include high-pressure mercury injection, nuclear magnetic resonance, micro CT, low-temperature nitrogen adsorption, and transmission electron microscope 
[14-20]. Due to the limitations of different measurement methods, the pore structure of coal can hardly be accurately characterized with a single method [21].

In previous studies, high-pressure mercury injection and low-temperature nitrogen adsorption are considered to be two effective methods for pore structure testing [22-24]. The principle of high-pressure mercury injection is to enable mercury to enter pores by applying an external force. When the external force is larger, mercury can enter pores with a smaller diameter. High-pressure mercury injection can measure pores whose diameters range from $5.5 \mathrm{~nm}$ to $1,000,00$ $\mathrm{nm}$. When the mercury injection pressure is higher than $200 \mathrm{MPa}$, mercury is able to enter pores with the diameter of $7 \mathrm{~nm}$. However, in this case, the excessive external force may pose the risk of deformation and damage to coal. Thus, this method cannot accurately measure the distribution of nanoscale pores [25].

The principle of low-temperature nitrogen adsorption is based on physical adsorption caused by intermolecular forces [26]. At $-195.8^{\circ} \mathrm{C}$, the energy of nitrogen molecules is reduced, and nitrogen molecules reach the adsorption equilibrium of approximate monolayer on the surface of solid material under the action of van der Waals forces. At this time, the adsorption surface area of the solid substance is proportional to its adsorption capacity. In this way, the specific surface area of the substance to be measured can be calculated. Meanwhile, with the volume of pores in the solid substance being equivalent to the volume of liquid nitrogen entering the pores, the rate of variation of pore volume with the pore size can be measured by means of theoretical analysis. The most commonly used calculation method is to calculate pore volume, pore size distribution, and pore specific surface area by using the Barrett-JoynerHalenda (BJH) theory [27]. Despite its inability to measure the distribution of macropores, low-temperature nitrogen adsorption is able to accurately characterize the distribution of micropores and small pores. Accordingly, some scholars propose to combine the above two methods for the purpose of accurately characterizing the full-size pore structure of coal [28-30].

Considering the important position of coal in China's energy structure, investigating the pore structure distribution characteristics of typical coal samples from different mining areas in China conduces to conducting an in-depth study on the control mechanism of pore structure on the gas adsorption, desorption, and seepage characteristics of coal. Such an investigation is of great significance for gas disaster prevention and CBM extraction in typical mining areas in China. In this paper, six different-rank coal samples were collected from different mining areas and then subjected to the high-pressure mercury injection experiment and the lowtemperature nitrogen adsorption experiment. Based on the experimental results, the mercury injection-ejection curves of different coal samples and their corresponding pore shapes, pore volumes, and pore specific surface areas were analyzed, and the low-temperature nitrogen adsorptiondesorption isotherms of these samples and their corresponding pore characteristics, pore volumes, and pore specific surface areas were also probed into. Moreover, the accuracy of these two methods in terms of measuring the pore volumes and pore specific surface areas under different pore diameters was discussed. Besides, the two methods were combined to characterize the full-size pore shapes, pore volumes, and pore specific surface areas of different coal samples. Ultimately, the influences and control mechanisms of coal rank on the pore structure characteristics were clarified.

\section{Materials and Methods}

2.1. Sampling Regions and Sample Preparation. The coal samples used in the experiment were selected from six different typical mining areas in China. The sampling regions are shown in Figure 1. Then, the water contents, ash contents, volatile contents, and vitrinite reflectance values of the samples were tested in accordance with the preparation process displayed in Figure 2. The sampling locations and the test results of relevant basic parameters are given in Table 1.

2.2. High-Pressure Mercury Injection Experiment. The device used in the high-pressure mercury injection experiment is the Auto Pore 9505 automatic mercury porosimeter in the Key Laboratory of Gas Geology and Gas Control of Henan Polytechnic University (Figure 3). The test pore diameter ranges from $5 \mathrm{~nm}$ to $360,000 \mathrm{~nm}$, and the maximum working pressure is $228 \mathrm{MPa}$. The application of pressure enables mercury to enter the pores. The functional relationship between the pore size that mercury can reach and the applied external force satisfies the Washburn equation [31, 32].

$$
r=\frac{-2 \gamma \cos \theta}{P}
$$

where $P$ is the mercury injection pressure, $\mathrm{Pa}$; $r$ is the radius of pores, $\mathrm{m} ; \theta$ is the contact angle, and it takes the value $130^{\circ} ; \gamma$ is the surface tension of mercury, and it equals $4.85 \times 10^{-3} \mathrm{~N} / \mathrm{m}$.

The specific surface area of pores in the sample can be further calculated via the mercury injection pressure according to the Young-Duper equation.

$$
-P d V=\gamma \cos \theta d S
$$

where $S$ is the specific surface area of pores, $\mathrm{m}^{2} / \mathrm{g}$.

2.3. Low-Temperature Nitrogen Adsorption Experiment. The device used in the low-temperature nitrogen adsorption experiment is the ASAP2020M automatic specific surface area and pore size analyzer in the Key Laboratory of Gas Geology and Gas Control of Henan Polytechnic University. The test pore size ranges from $0.35 \mathrm{~nm}$ to $500 \mathrm{~nm}$, and the lower limit of specific surface area measurement is 0.0005 $\mathrm{m}^{2} / \mathrm{g}$. The schematic diagram of the low-temperature nitrogen adsorption experimental device is shown in Figure 4.

In the experiment, the processed sample was put into the sample tube before the experimental device was connected, and then, the liquid nitrogen cup was filled with liquid nitrogen. Next, the entire system was vacuumized, and the volume of free space in the sample tube was calibrated using helium. After a certain amount of nitrogen was injected into the 


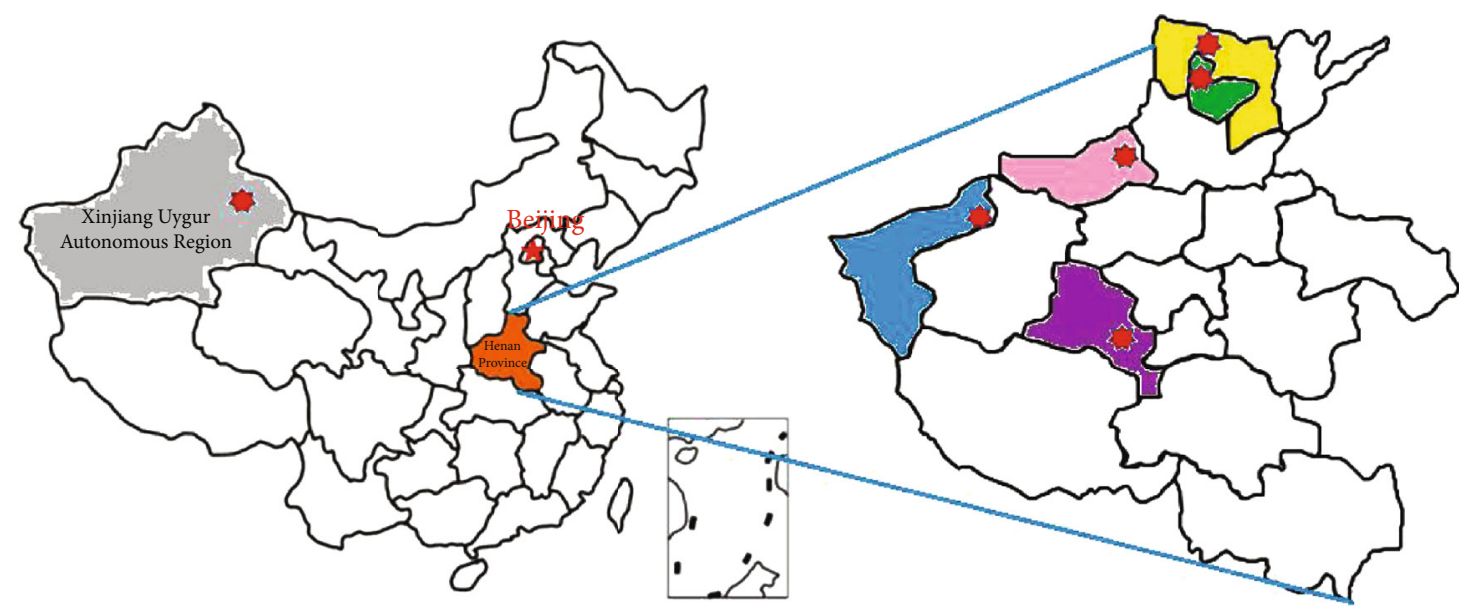

China

Henan Province

Beijing

Sample 1

Sample 2

Sample 3

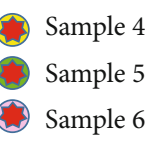

Sample 4
Sample 5
Sample 6

FIgURE 1: Sampling locations of experimental coal samples.

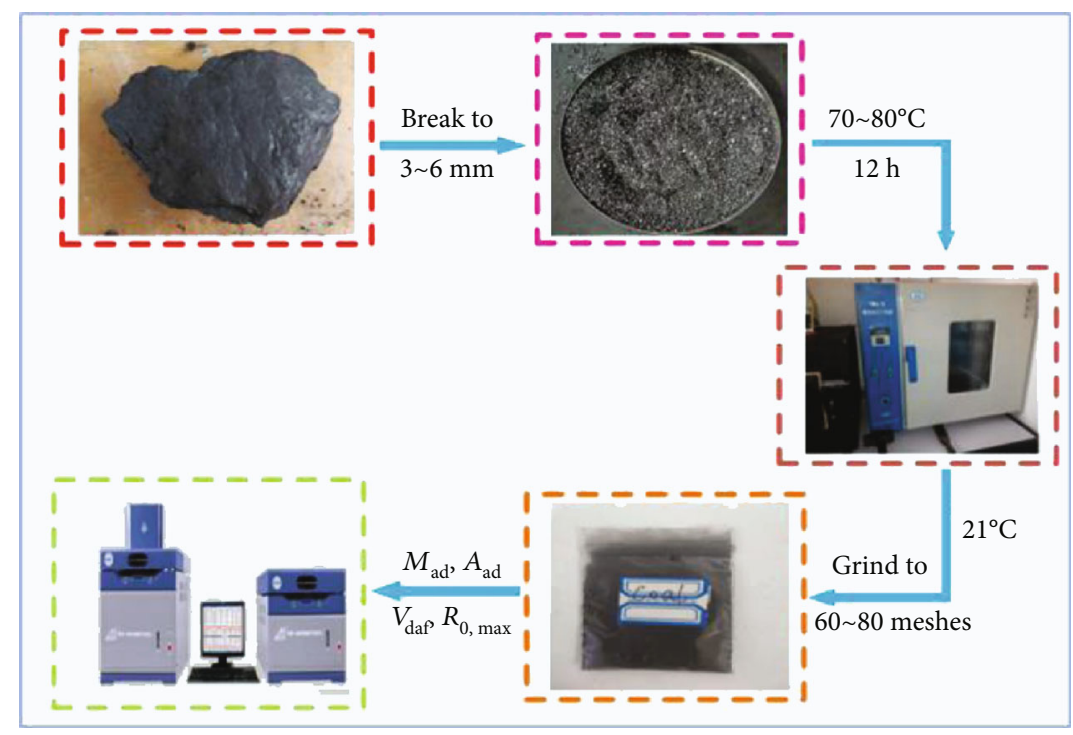

FIGURE 2: Sample preparation process and experimental steps.

TABLE 1: Basic information and parameters of experimental coal samples.

\begin{tabular}{|c|c|c|c|c|c|}
\hline Sample & Sampling location & $M_{\mathrm{ad}}(\%)$ & $A_{\text {ad }}(\%)$ & $V_{\text {daf }}(\%)$ & $R_{0, \max }(\%)$ \\
\hline Sample 1 & Dananhu No. 1 mine, Hami mining area & 8.02 & 15.36 & 42.35 & 0.43 \\
\hline Sample 2 & Gengcun mine, Yima mining area & 2.11 & 11.92 & 38.16 & 0.52 \\
\hline Sample 3 & Pingdingshan No. 5 mine, Pingdingshan mining area & 1.23 & 15.82 & 28.82 & 1.05 \\
\hline Sample 4 & Zhujiao mine, Anyang mining area & 1.62 & 11.85 & 22.16 & 1.30 \\
\hline Sample 5 & Hebi No. 9 mine, Hebi mining area & 1.37 & 12.86 & 12.93 & 1.76 \\
\hline Sample 6 & Jiulishan mine, Jiaozuo mining area & 2.32 & 12.92 & 7.68 & 2.55 \\
\hline
\end{tabular}

Note: $M_{\mathrm{ad}}, A_{\mathrm{ad}}$, and $V_{\mathrm{daf}}$ are the moisture, ash, and volatile matter contents on the air-dried basis, respectively. $R_{0, \max }$ is the maximum vitrinite reflectance. 


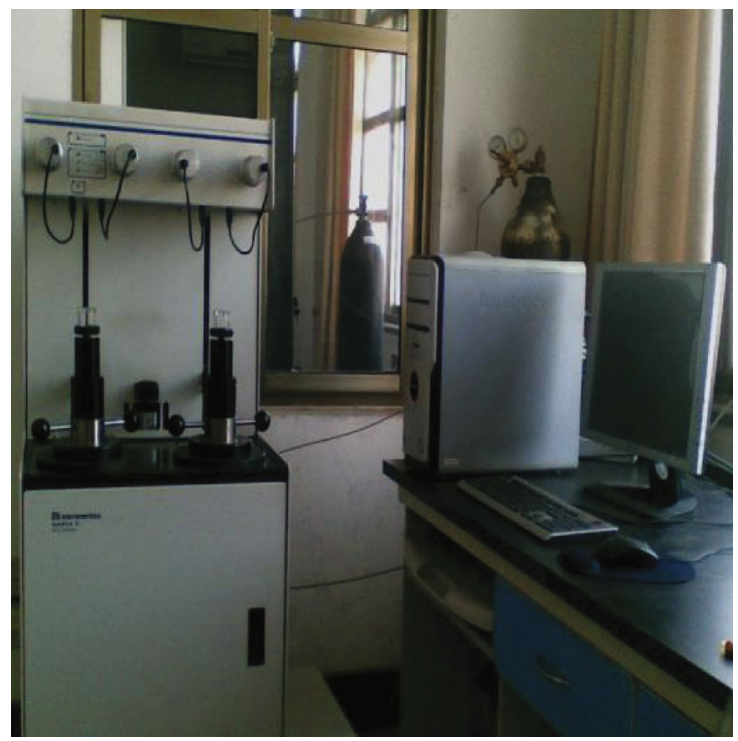

FIgURe 3: Auto Pore 9505 automatic mercury porosimeter.

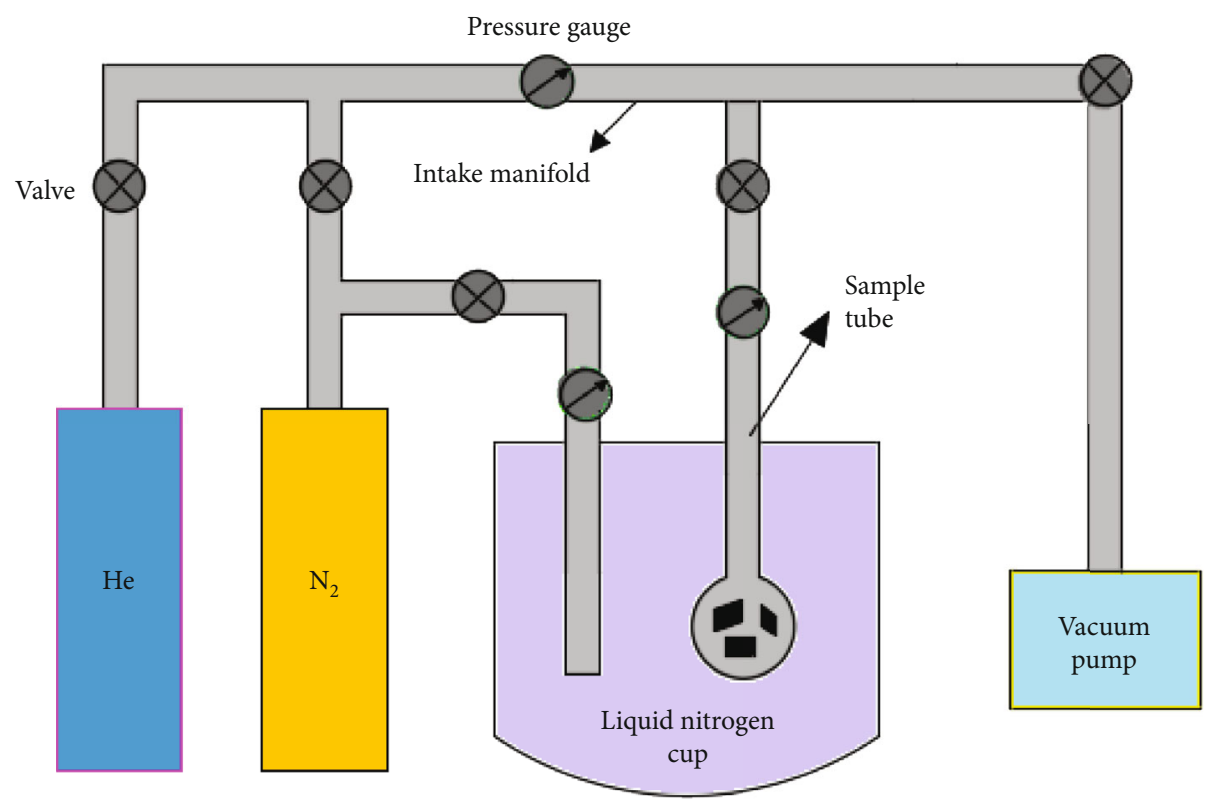

FIgURE 4: Schematic diagram of the low-temperature nitrogen adsorption experimental device.

intake manifold, the initial pressures, initial temperatures, and initial gas weights of the intake manifold and the sample tube were recorded. Afterwards, a certain amount of nitrogen was injected from the intake manifold to the sample tube. When the sample reached adsorption equilibrium, the pressures, temperatures, and gas weights of the intake manifold and the sample tube were recorded again. On the basis of the acquired data, the adsorption capacity of the sample was calculated. Then, the nitrogen adsorption-desorption isotherm can be obtained by taking the relative pressure as the abscissa and the adsorption capacity as the ordinate.

Generally, the pore volume, pore size distribution, and the specific surface area are calculated by using the $\mathrm{BJH}$ method [33].

\section{Experimental Results}

\subsection{Results of the High-Pressure Mercury Injection Experiment}

3.1.1. Pore Volume and Pore Specific Surface Area. The pore volumes and pore specific surface areas of the six coal samples were tested by performing a high-pressure mercury injection experiment. The results are exhibited in Figure 5.

The pores in the six different-rank coal samples mainly belong to small pores and micropores, followed by macropores and mesopores, as displayed in Figures 5(a) and 5(b). Besides, the total pore volume decreases first and then increases with the rise of coal rank, as illustrated in Figure 5(e). 


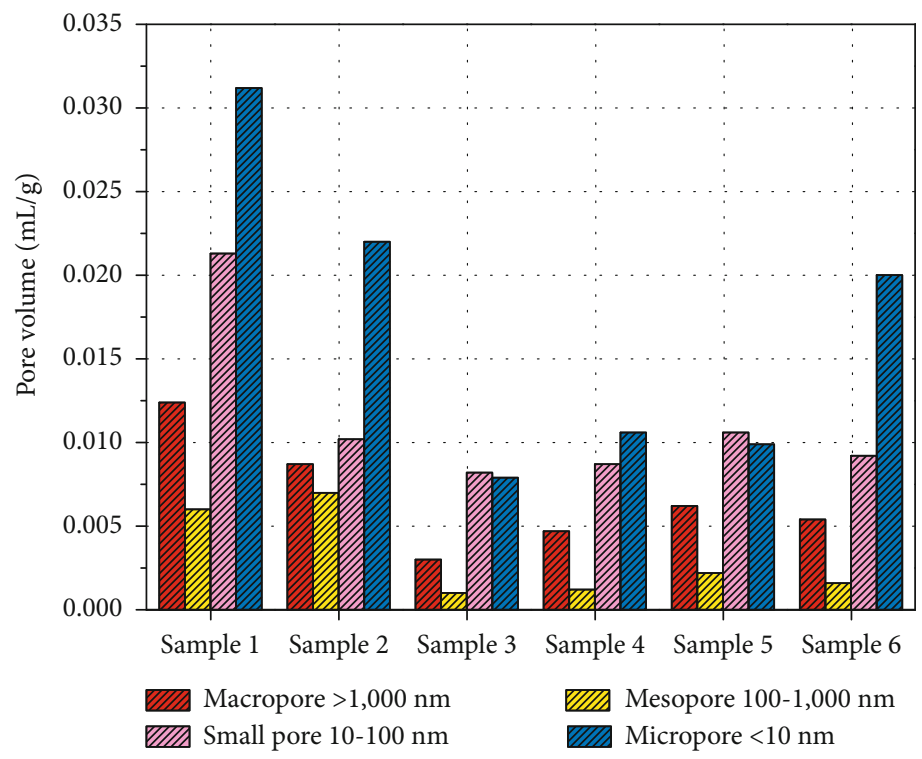

(a)

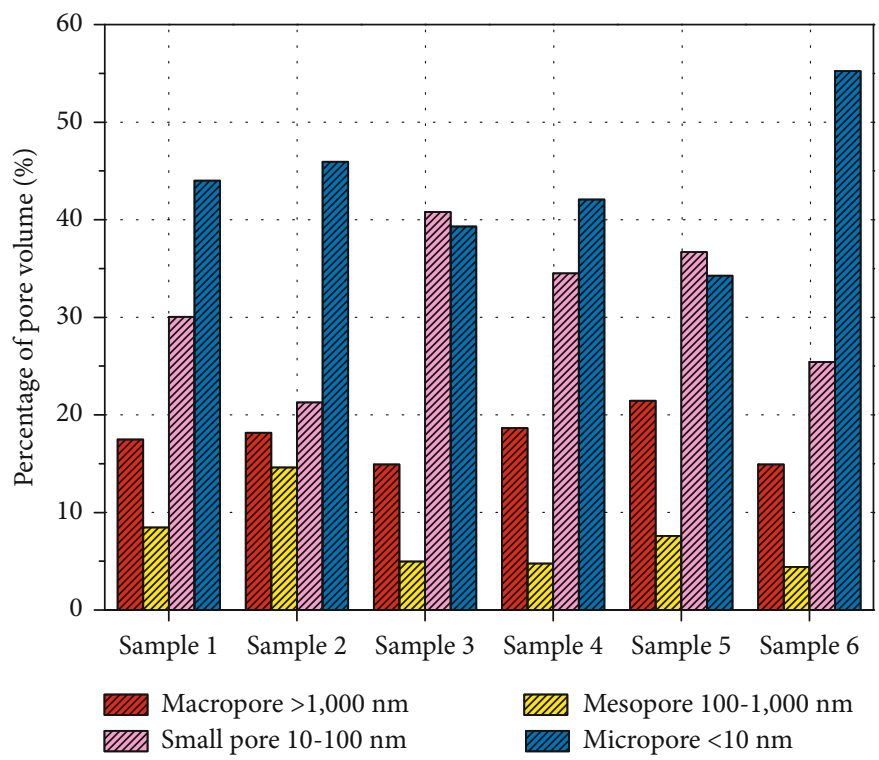

(b)

Figure 5: Continued. 


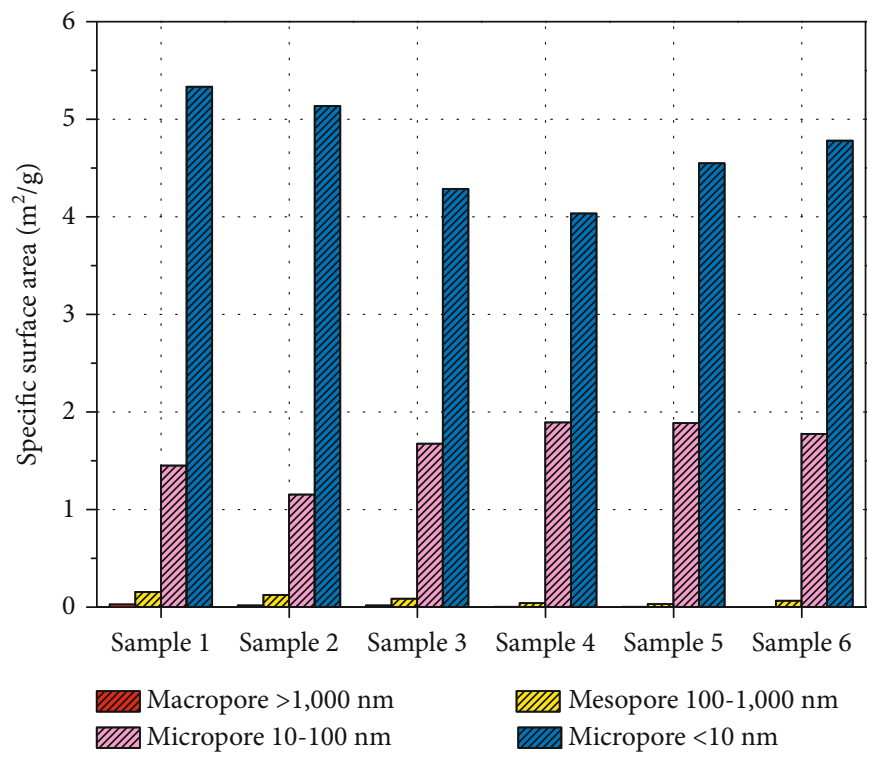

(c)

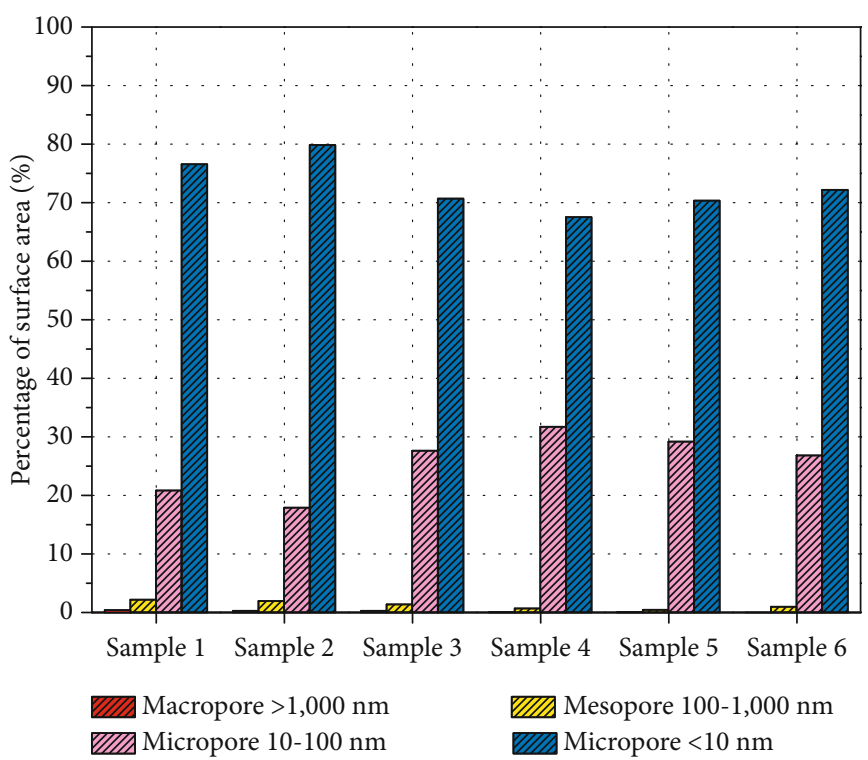

(d)

Figure 5: Continued. 


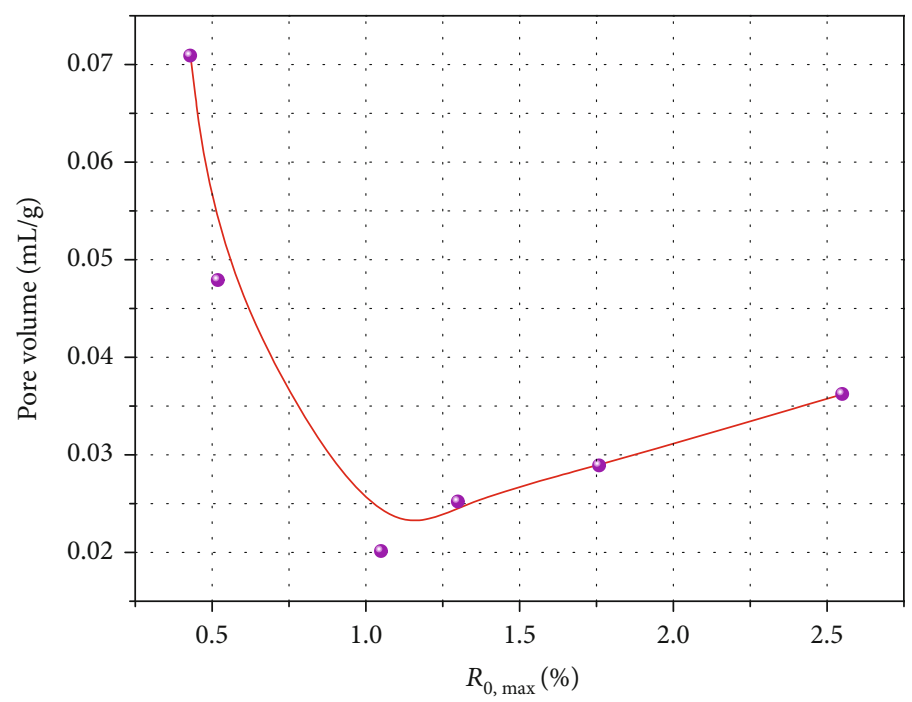

(e)

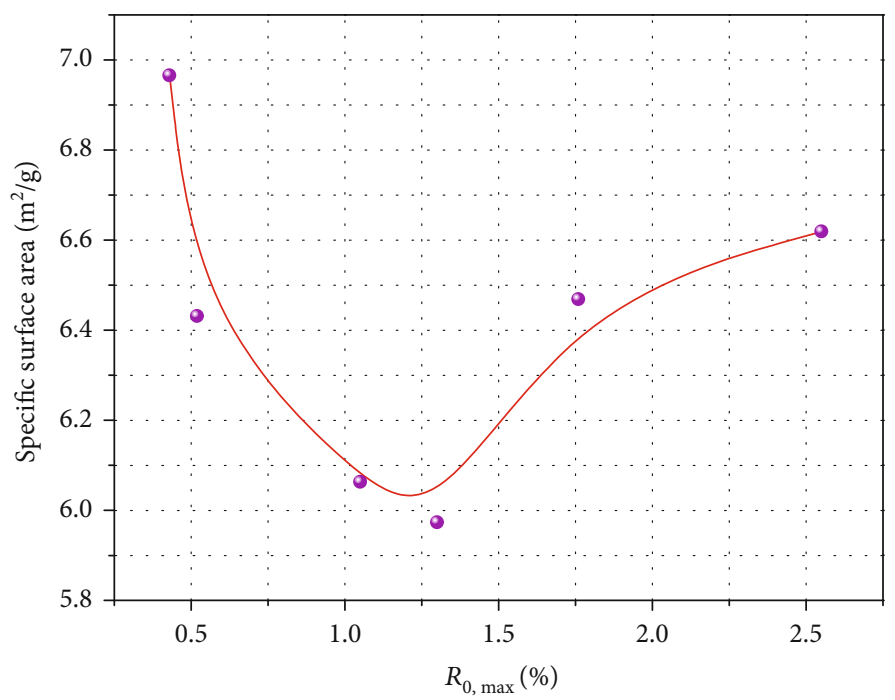

(f)

FIgURE 5: Results of the high-pressure mercury injection experiment. (a) Pore volume distribution; (b) pore volume proportion; (c) pore specific surface area distribution; (d) pore specific surface area proportion; (e) shows the relationship between total pore volume and metamorphic degree; (f) shows the relationship between total specific surface area and metamorphic degree.

Meanwhile, the pore specific surface areas of micropores account for the largest proportion (over 67.57\%) for all the six coal samples, followed by those of small pores, whereas those of macropores and mesopores account for relatively smaller proportions, as presented in Figures 5(c) and 5(d). Hence, it can be concluded that the total pore specific surface areas of coal samples are primarily determined by micropores, and the total specific surface area decreases first and then increases with the rise of coal rank, as illustrated in Figure 5(f).

3.1.2. Mercury Injection Curve and Pore Shape Characteristics. The pores in coal can be divided into effective pores and isolated pores. The effective pores can be further divided into semiclosed pores, open pores, and ink-bottle-shaped pores. The pore shapes are illustrated in Figure 6 [34].

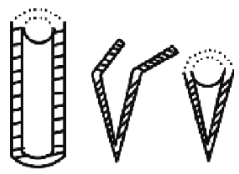

(a) Semiclosed pore

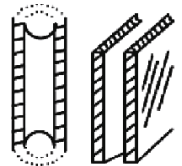

(b) Open pore

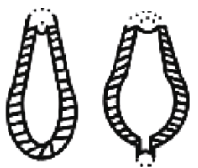

(c) Ink-bottle-shaped pore
FIgURE 6: Classification of pore shapes corresponding to the mercury injection curves.

The mercury injection-ejection curves obtained from the high-pressure mercury injection experiment are shown in Figure 7. The mercury injection-ejection curves of different samples differ obviously. Although hysteresis loops are formed by the curves of all the six samples, these hysteresis loops are quite different, which reflects the differences in 

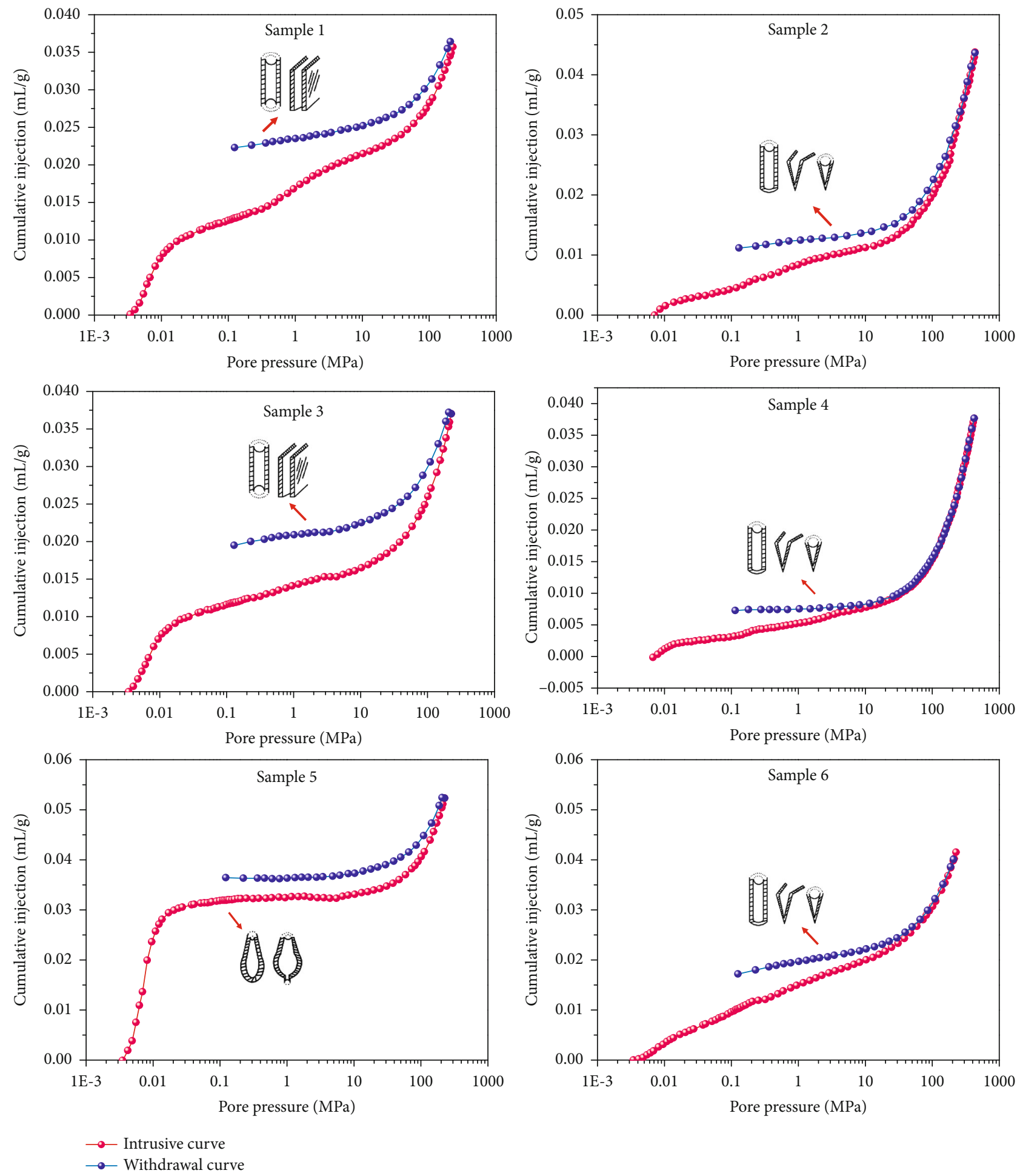

FiguRE 7: Mercury injection curves of experimental coal samples.

the basic pore shapes and connectivities of different coal samples. According to the classification method of pore shapes in Figure 6, the six coal samples can be divided into 3 categories:

(1) Samples 1 and 3. Their values of mercury injectionejection curves differ notably, and obvious hysteresis loops are formed by their curves, which suggests that the two samples possess open pores and a certain number of ink-bottle-shaped pores. In addition, the cumulative amounts of mercury injection of the two samples increase slowly with the rise of pressure, which demonstrates the development of differentsized pores in the samples. 


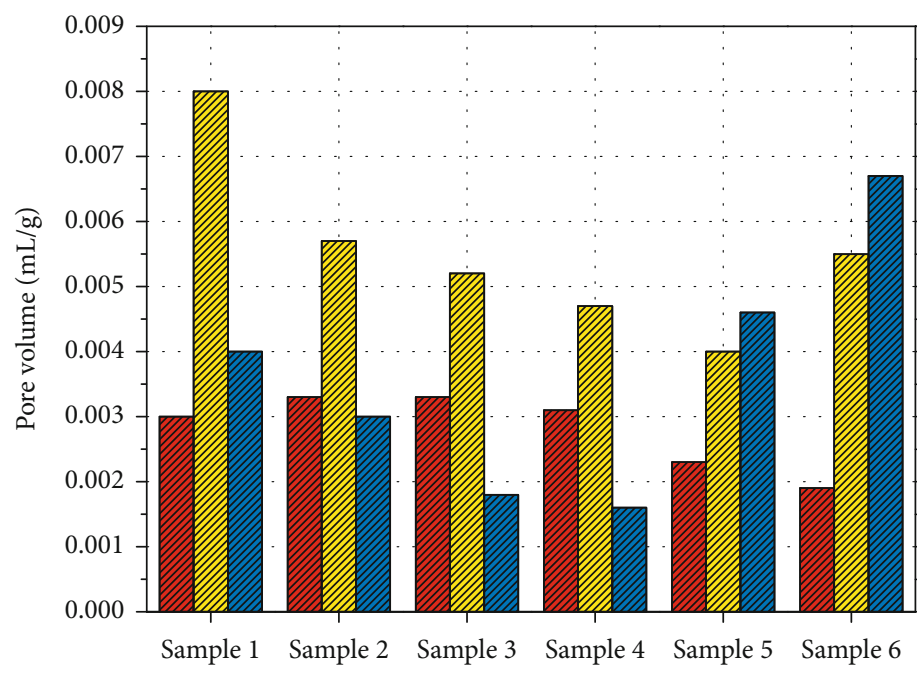

IIIA Mesopore 100-1,000 nm

Micropore $<10 \mathrm{~nm}$

प1IA Small pore $10-100 \mathrm{~nm}$

(a)

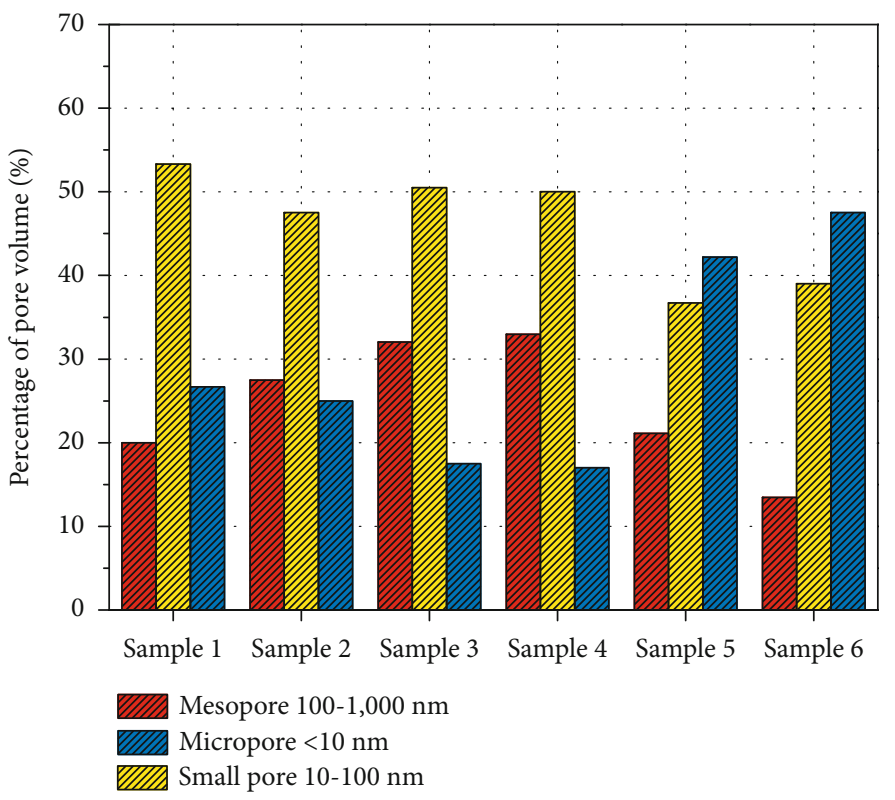

(b)

Figure 8: Continued. 


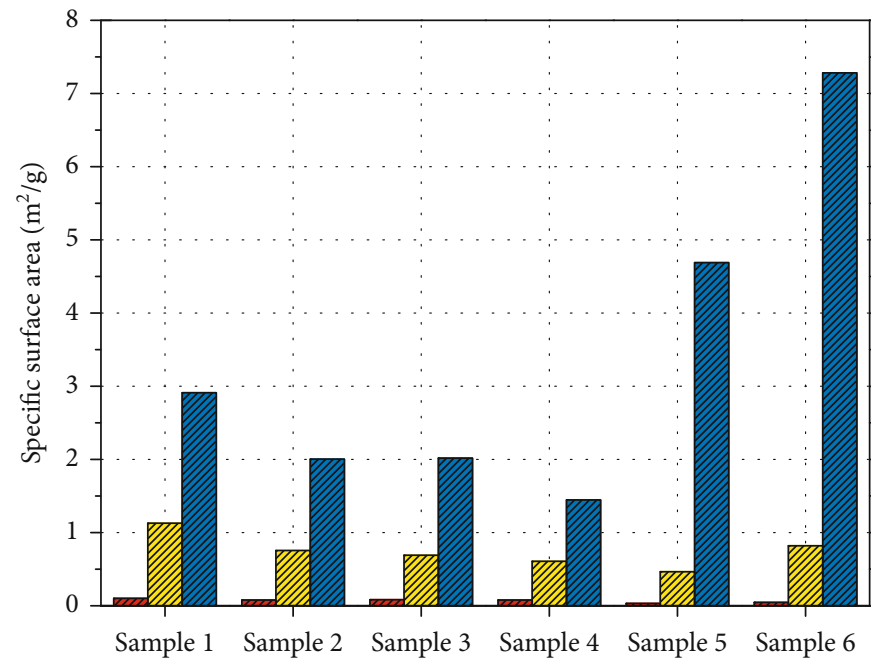

IIIA Mesopore 100-1,000 nm

Micropore $<10 \mathrm{~nm}$

पIIIA Small pore $10-100 \mathrm{~nm}$

(c)

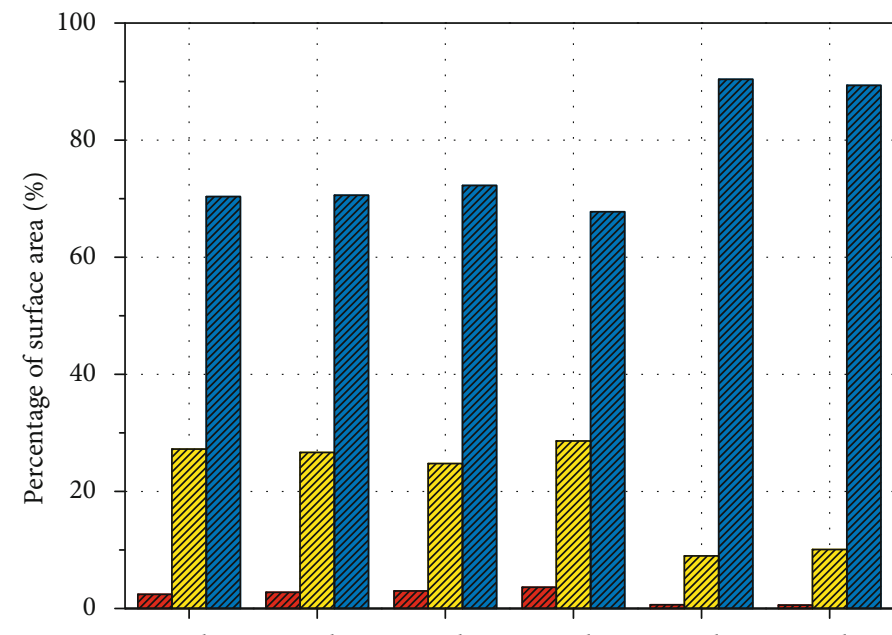

Sample 1 Sample 2 Sample 3 Sample 4 Sample 5 Sample 6

Mesopore 100-1,000 nm

IIIA Micropore $<10 \mathrm{~nm}$

VIII Small pore $10-100 \mathrm{~nm}$

(d)

Figure 8: Continued. 


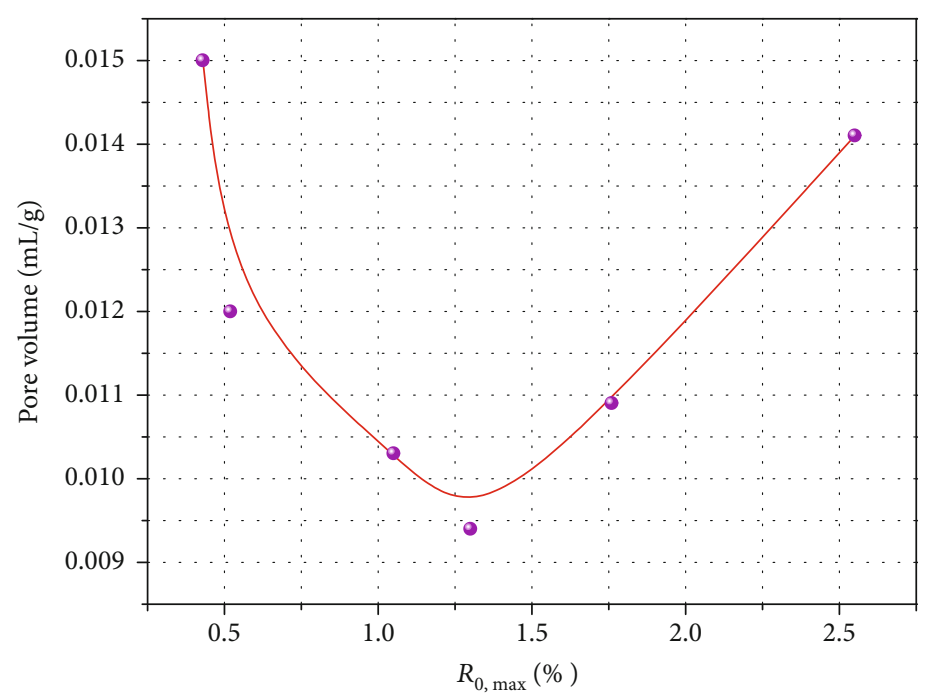

(e)

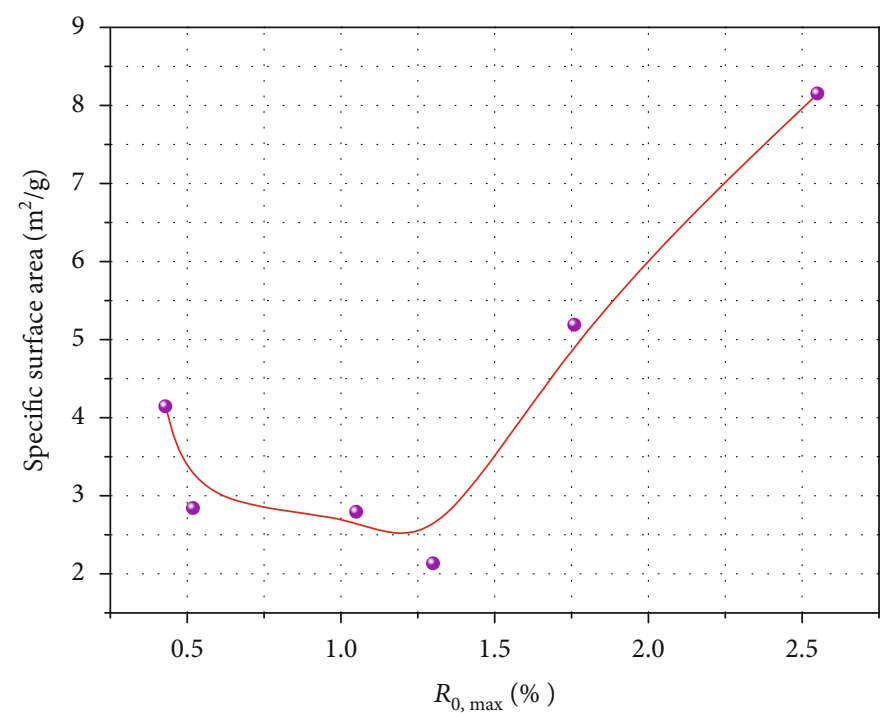

(f)

FIGURE 8: Results of the low-temperature nitrogen adsorption experiment. (a) Pore volume distribution; (b) pore volume proportion; (c) pore specific surface area distribution; (d) pore specific surface area proportion; (e) shows the relationship between total pore volume and metamorphic degree; (f) shows the relationship between total specific surface area and metamorphic degree.

(2) Sample 5. When the pressure is lower than $0.01 \mathrm{MPa}$, its cumulative pore volume jumps sharply, indicating the wide development of large pores. When the pressure is higher than $10 \mathrm{MPa}$, its cumulative pore volume grows rapidly, suggesting the wide development of micropores. Besides, a hysteresis loop is formed by the curve, which is a sign of the existence of inkbottle-shaped pores.

(3) Samples 2, 4, and 6. The hysteresis loops of the three samples are the smallest, and they even disappear when the relative pressure gets higher than $10 \mathrm{MPa}$, which shows that the pores in the three samples are not so open and gradually close in the later stage. This demonstrates that micropores and transition pores are semiclosed. Meanwhile, the samples con- tain a large number of semiopen pores whose connectivities are poor.

\subsection{Results of the Low-Temperature Nitrogen Adsorption Experiment}

3.2.1. Pore Volume and Pore Specific Surface Area. The pore volumes and pore specific surface areas of the six coal samples were tested through a low-temperature nitrogen adsorption experiment. The results are exhibited in Figure 8.

The pores in the six different-rank coal samples mainly belong to small pores and micropores, followed by macropores and mesopores, as displayed in Figures 8(a) and 8(b). In addition, the total pore volume decreases first and then increases with the rise of coal rank, as illustrated in Figure 8(e). 
Meanwhile, the pore specific surface areas of micropores account for the largest proportion (over 67.57\%) for all the six coal samples, whereas those of mesopores and small pores account for relatively smaller proportions, as presented in Figures $8(\mathrm{c})$ and $8(\mathrm{~d})$. Hence, it can be concluded that the total pore specific surface areas of coal samples are mainly determined by micropores, and the total specific surface area decreases first and then increases with the rise of coal rank, as illustrated in Figure 8(f).

\subsubsection{Adsorption Isotherms and Pore Shape Characteristics.} The shape of adsorption isotherm obtained through the low-temperature nitrogen adsorption experiment can reflect the pore shape. According to whether an adsorption loop is formed by the nitrogen adsorption isotherm, Chen and Tang [35] divided the pore shapes into parallel plate pores, cylindrical pores, and ink-bottle-shaped pores. The pore shapes are exhibited in Figure 9.

Figure 10 shows the nitrogen adsorption isotherms of the six coal samples. The adsorption capacities of different samples differ significantly. To be specific, the maximum adsorption capacities of samples $1-6$ are $1.569 \mathrm{~cm}^{3} / \mathrm{g}, 0.762$ $\mathrm{cm}^{3} / \mathrm{g}, 7.135 \mathrm{~cm}^{3} / \mathrm{g}, 8.668 \mathrm{~cm}^{3} / \mathrm{g}, 8.055 \mathrm{~cm}^{3} / \mathrm{g}$, and 10.566 $\mathrm{cm}^{3} / \mathrm{g}$, respectively. Among them, sample 2 has the smallest adsorption capacity while sample 6 has the largest adsorption capacity. The adsorption capacity varies in a U-shaped trend, i.e., it decreases first and then increases, with the rise of coal rank.

Based on the nitrogen adsorption isotherms, the six coal samples can be classified into the following three categories in accordance with the pore shapes given in Figure 9.

(1) Samples 1, 2, 5, and 6. Their adsorption isotherms and desorption isotherms are separated, not overlapping even at a low relative pressure. This shows that the micropores are relatively developed in the four samples, and most of the pores belong to parallel plate pores with four open edges. In the adsorption process, a turning point which corresponds to the ink-bottle-shaped pores appears on the desorption isotherm of sample 1 at the relative pressure of about 0.5 .

(2) Sample 3. Its adsorption isotherms and desorption isotherms roughly overlap, indicating that it primarily consists of cylindrical pores with one closed end. Since such pores require equal relative pressures during adsorption and desorption, the adsorption isotherms and the desorption isotherms are basically not separated.

(3) Sample 4. Its adsorption isotherms and desorption isotherms do not completely overlap in the whole nitrogen adsorption process. Instead, they merely roughly overlap at relatively low pressures and high relative pressures, indicating the existence of open permeable and impermeable pores in the sample. Moreover, a turning point which corresponds to the ink-bottle-shaped pores appears at the relative pressure of about 0.5 .

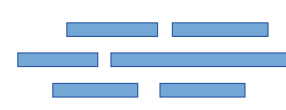

(a) Parallel plate pore

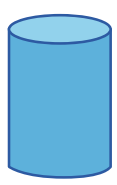

(b) Cylindrical pore

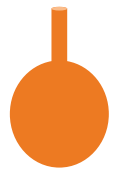

(c) Ink-bottle-typed pore
FIgURE 9: Pore shapes corresponding of the adsorption isotherms.

3.3. Joint Characterization of Pore Volume. The volumes of mesopores and macropores can be accurately tested through the high-pressure mercury injection experiment, while those of micropores and small pores can be accurately determined through the low-temperature nitrogen adsorption experiment. Thus, in this study, the two experiments were combined to jointly characterize the full-size pore volumes of the six experimental coal samples.

The principle of joint characterization of full-size pore characteristics is to take the mesopore and macropore volume data from the high-pressure mercury injection experiment and the micropore and small pore data from the lowtemperature nitrogen adsorption experiment as the full-size pore volumes of the coal samples. The joint characterization results are listed in Table 2.

The total pore volumes of the experimental six coal samples lie in the range of $0.0115-0.0284 \mathrm{~mL} / \mathrm{g}$. Among them, sample 1 has the largest pore volume and sample 3 has the smallest one. The total pore volume varies in a U-shaped trend, i.e., it decreases first and then increases, with the increase of coal rank. Specifically, with respect to macropores and mesopores, Sample 1 and sample 3 correspond to the largest and smallest pore volumes, respectively. With respect to small pores, sample 1 and sample 6 correspond to the largest and smallest pore volumes, respectively. With respect to micropores, sample 6 and sample 4 correspond to the largest and smallest pore volumes, respectively. In the measured data, micropores share a relatively consistent variation trend with the total pore volume.

3.4. Joint Characterization of Pore Specific Surface Area. According to the principle of joint characterization given in Section 3.3, the pore specific surface areas of the six coal samples are characterized. The characterization results are listed in Table 3.

The total pore specific surface areas of the six experimental coal samples lie in the range of $2.099-8.168 \mathrm{~m}^{2} / \mathrm{g}$. Among them, sample 4 has the smallest pore specific surface area and sample 6 has the largest one. The total pore specific surface area also varies in an approximately $\mathrm{W}$-shaped trend with the rise of coal rank. The pore specific surface areas of all the six coal samples present the following variation trend: micropores $>$ small pores $>$ mesopores $>$ macropores. In addition, under the condition of the same coal rank, the pore specific surface areas of small pores and micropores are much larger than those of mesopores and macropores. This means that mesopores and macropores contribute little to the pore specific surface area of coal, while micropores are the main contributor to it. Generally, the coal with a larger total pore 

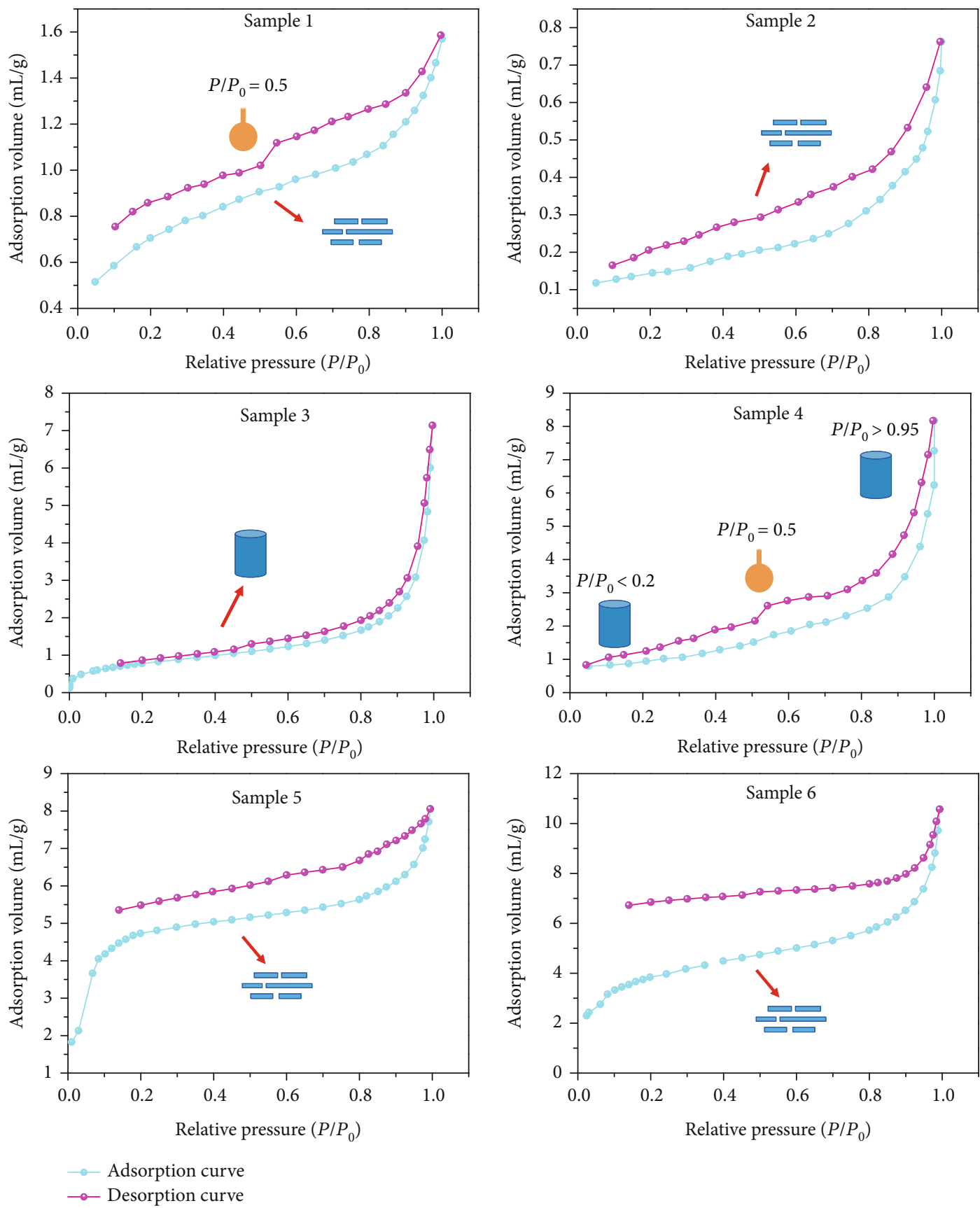

FIGURE 10: Nitrogen adsorption isotherms of experimental coal samples.

specific surface area boasts stronger adsorption capacity. Hence, micropores are the main controlling factor that determines the adsorption capacity of coal.

\section{Discussion}

4.1. Effect of Metamorphic Degree on Pore Volume. In this section, correlation analysis was conducted on the pore volumes and volatile matter contents of the six coal samples after joint characterization. Through fitting, it is found that the relationship between pore volume and volatile matter content conforms to a binomial relationship. For all the six coal samples, the pore volume varies in a trend resembling a reverse parabola with the increase of the volatile matter content (Figure 11).

Based on the correlation analysis on the results of fitting between pore volume and volatile matter content, the following conclusions can be drawn.

(1) The fitting degrees $R^{2}$ of macropores, mesopores, and micropores are all above 0.75 , which means that the fitting curves can well reflect the relationship between volatile matter content and pore volume. In the stage of low metamorphic degree $\left(V_{\mathrm{daf}}>25 \%\right)$, the volumes 
TABLE 2: Joint characterization results of pore volume.

\begin{tabular}{lccccc}
\hline \multirow{2}{*}{ Sample } & & \multicolumn{2}{c}{ Pore volume $(\mathrm{mL} / \mathrm{g})$} & & \multirow{2}{*}{ Total pore volume $(\mathrm{mL} / \mathrm{g})$} \\
\hline Sample 1 & Macropore & Mesopore & Small pore & Micropore & 0.0284 \\
Sample 2 & 0.0124 & 0.0040 & 0.0080 & 0.0040 & 0.0204 \\
Sample 3 & 0.0087 & 0.0030 & 0.0057 & 0.0030 & 0.0110 \\
Sample 4 & 0.0030 & 0.0010 & 0.0052 & 0.0018 & 0.0122 \\
Sample 5 & 0.0047 & 0.0012 & 0.0047 & 0.0016 & 0.0170 \\
Sample 6 & 0.0062 & 0.0022 & 0.0040 & 0.0046 & 0.0202 \\
\hline
\end{tabular}

TABLE 3: Joint characterization results of pore specific surface area.

\begin{tabular}{lccccc}
\hline \multirow{2}{*}{ Sample } & & \multicolumn{2}{c}{ Specific surface area $\left(\mathrm{m}^{2} / \mathrm{g}\right)$} & Total specific surface area $\left(\mathrm{m}^{2} / \mathrm{g}\right)$ \\
\hline Sample 1 & Macropore & Mesopore & Small pore & Micropore & 4.221 \\
Sample 2 & 0.027 & 0.154 & 1.127 & 2.913 & 2.905 \\
Sample 3 & 0.017 & 0.126 & 0.757 & 2.005 & 2.810 \\
Sample 4 & 0.017 & 0.085 & 0.691 & 2.017 & 2.099 \\
Sample 5 & 0.003 & 0.042 & 0.610 & 1.444 & 5.188 \\
Sample 6 & 0.002 & 0.030 & 0.467 & 4.689 & 8.168 \\
\hline
\end{tabular}

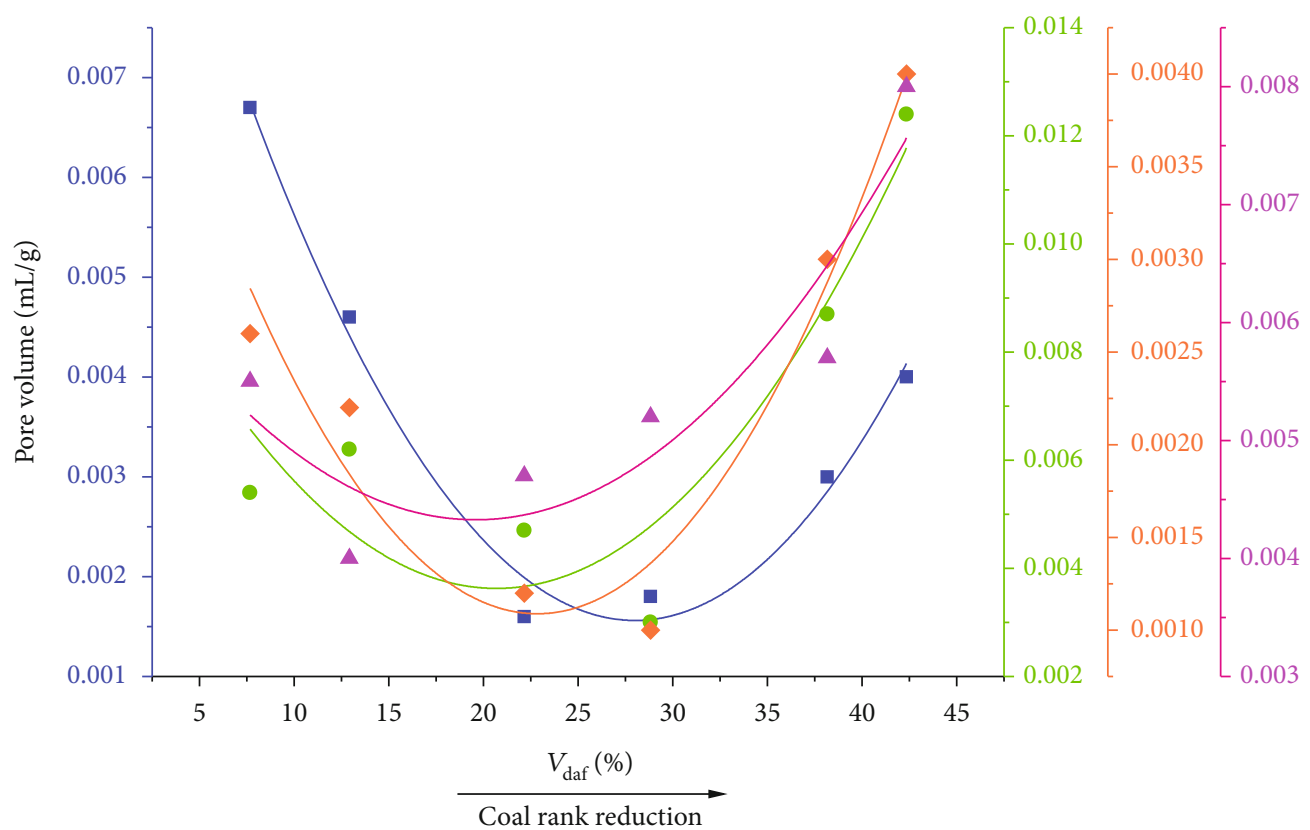

$$
\begin{array}{ll}
\text { - Macropore } & -R^{2}=0.75175 \\
\text { - Mesopore } & -R^{2}=0.90936 \\
\text { - Small pore } & -R^{2}=0.73482 \\
\text { - Micropore } & -R^{2}=0.97427
\end{array}
$$

FIGURE 11: Curves of fitting between volatile matter content and pore volume.

of various pores all surge notably with the increase of volatile matter content. In the stage of medium metamorphic degree $\left(15 \%<V_{\text {daf }}<25 \%\right)$, they are rarely affected by the volatile matter content, just changing slightly. In the stage of high metamorphic degree $\left(V_{\mathrm{daf}}<15 \%\right)$, they drop considerably with the increase of volatile matter content
(2) The fitting degree $R^{2}$ of small pores is 0.73 , which means that the fitting curves can roughly reflect relationship between volatile matter content and pore volume. The pore volume grows continuously with the increase of volatile matter content, indicating that the volume of small pores is greatly affected by the volatile matter content 


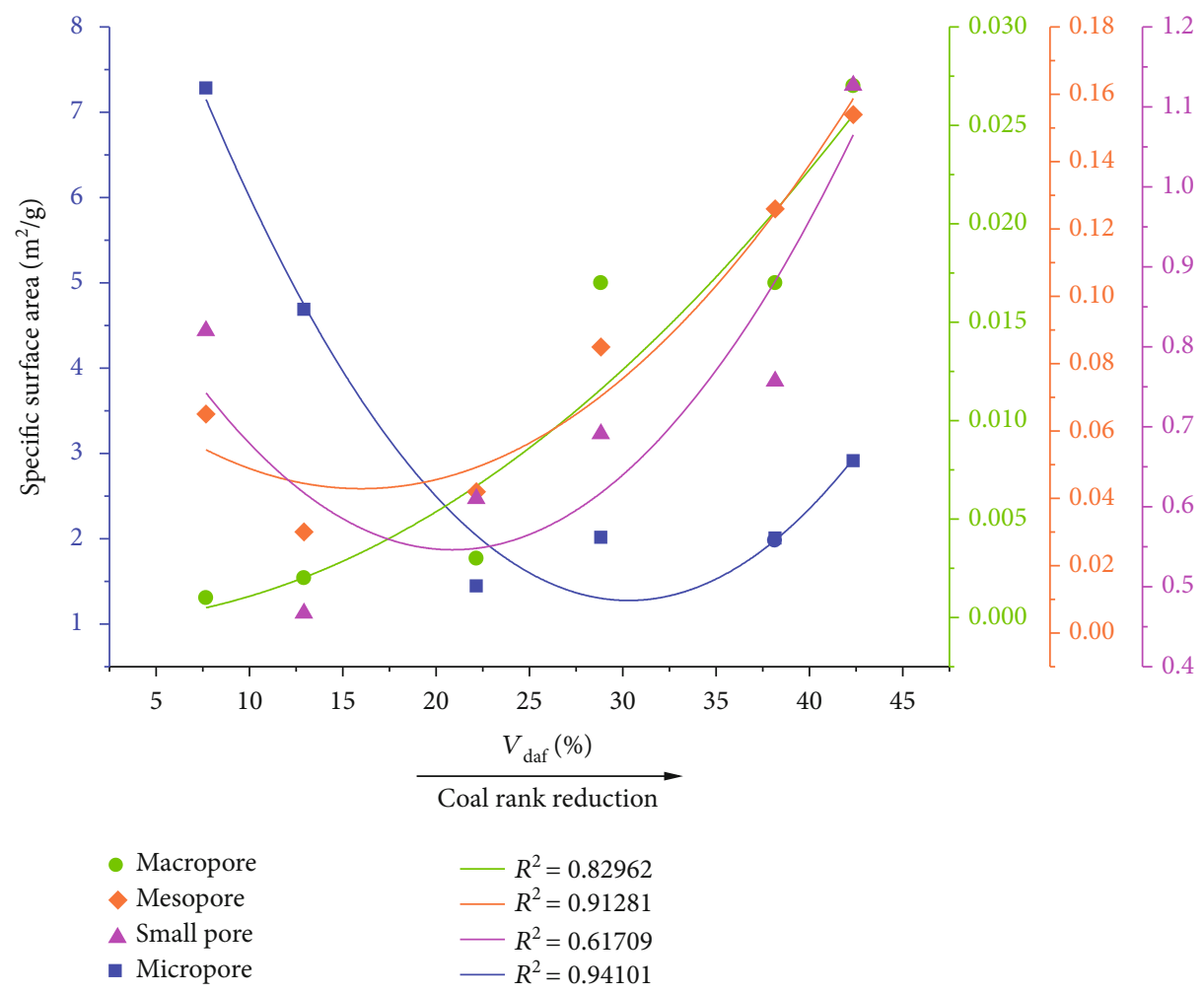

Figure 12: Curve of fitting between volatile matter and pore specific surface area.

4.2. Effect of Metamorphic Degree on Pore Specific Surface Area. In this section, correlation analysis was conducted on the pore specific surface areas and volatile matter contents of the six coal samples after joint characterization. Through fitting, it is disclosed that the relationship between pore specific surface area and volatile matter content conforms to a binomial relationship. For all the six coal samples, the pore specific surface area also varies in a trend resembling a reverse parabola with the increase of the volatile matter content (Figure 12).

Based on the correlation analysis on the results of fitting between pore specific surface area and volatile matter content, the conclusions are as follows.

(1) The fitting degrees $R^{2}$ of macropores and mesopores are both higher than 0.8 , which means that the relationship between volatile matter content and pore specific surface area is well reflected. With the increase of volatile matter content, the fitting curves of specific surface areas of macropores and mesopores present an upward trend, which demonstrates that the numbers of macropores and mesopores grow gradually

(2) The fitting degree $R^{2}$ of small pores is 0.62 , which means that the relationship between volatile matter content and pore specific surface area is roughly reflected. In the stage of low metamorphic degree, the pore specific surface area surges sharply, indicating the rapid growth of the number of small pores. In the stage of medium metamorphic degree, it is barely affected by the volatile matter content, and the number of small pores changes slightly. The stage of high metamorphic degree witnesses the rapid decreases of pore specific surface area and number of small pores

(3) The fitting degree $R^{2}$ of micropores is 0.94 , which means that the relationship between volatile matter content and pore specific surface area is excellently reflected. In the stage of medium metamorphic degree, the pore specific surface area changes insignificantly, suggesting the small change of the number of micropores. In the stages of low and high metamorphic degrees, it changes sharply, showing the great change of the number of micropores

\section{Conclusions}

In this study, the pore size, pore size distribution, and pore specific surface area of six different-rank coal samples from typical mining areas in China were calculated based on the results of high-pressure mercury injection and lowtemperature liquid nitrogen adsorption experiments. Furthermore, the full-size pore characteristic parameters of coal samples were jointly characterized by the two methods. Finally, the influences of metamorphic degree on pore structure were discussed. The following main conclusions were drawn:

(1) The results of the high-pressure mercury injection experiment and the low-temperature nitrogen adsorption experiment on the six coal samples reveal that the 
specific surface area of micropores accounts for the largest proportion, which indicates that micropores determine the adsorption capacities of coal samples and are the main space for gas adsorption. Besides, with the increase of metamorphic degree, the pore volumes and pore specific surface areas of the six coal samples all decrease first and then increase

(2) The results of the two experiments also disclose that the pore shape characteristics of the six coal samples differ notably. Such differences are a proof of the heterogeneity of surface properties of coal samples

(3) According to the results of joint characterization, the volumes and specific surface areas of micropores, small pores, mesopores, and macropores exhibit varying variation trends with the change of metamorphic degree. Specifically, the pore volume varies in a U-shaped trend while the pore specific surface area changes in an approximately U-shaped trend with the increase of coal rank

(4) The fitting degrees of volatile matter content and pore volume and those of volatile matter content and pore specific surface area are all greater than 0.6. The fitting curves can well reflect the influences and controlling mechanisms of metamorphic degree on pore volumes and pore specific surface areas of coal samples. With the increase of volatile matter content, the pore volume and the pore specific surface area both vary in a trend resembling a reverse parabola

\section{Data Availability}

The data used to support the findings of this study are included within the article.

\section{Conflicts of Interest}

The authors declare that they have no conflicts of interest.

\section{Acknowledgments}

This work was supported by the National Natural Science Foundation of China (Grant No.: 51974109), the Program for Leading Talents in Scientific and Technological Innovation of Henan Province (Grant No.: 204200510032), the Scientific and Technological Projects of Henan Province (Grant No.: 202102310220), the Plan of Key Scientific Research Project of Colleges and Universities in Henan Province (Grant No.: 20A620001), the Fundamental Research Funds for the Universities of Henan Province (Grant No.: NSFRF210301), and the Doctoral Fund of Henan Polytechnic University (Grant No.: B2019-55).

\section{References}

[1] X. Z. Wang, "Strategic consideration of China coal industry development during energy revolution and new normal of economic development," China Coal, vol. 41, no. 4, pp. 5-8, 2015.
[2] L. Yuan, N. Zhang, J. G. Han, and Y. Wang, "The concept, model and reserve forecast of green coal resources in China," Journal of China University of Mining and Technology, vol. 47, no. 1, pp. 1-8, 2018.

[3] F. Wu and Z. M. Zhao, "The development status and future forecast of China's coal supply," China Coal, vol. 44, no. 7 , pp. 5-8, 2018.

[4] F. Du, K. Wang, X. Zhang, C. P. Xin, L. Y. Shu, and G. D. Wang, "Experimental study of coal-gas outburst: insights from coal-rock structure, gas pressure and adsorptivity," Natural Resources Research, vol. 29, no. 4, pp. 2481-2493, 2020.

[5] Y. P. Yang, Z. H. Wen, L. L. Si, and X. Y. Xu, "Experimental study on variation law of electrical parameters and temperature rise effect of coal under DC electric field," Scientific Reports, vol. 11, no. 1, pp. 1-11, 2021.

[6] L. L. Si, H. T. Zhang, J. P. Wei, B. Li, and H. K. Han, "Modeling and experiment for effective diffusion coefficient of gas in water-saturated coal," Fuel, vol. 284, article 118887, 2021.

[7] H. J. Zhang, L. Zhang, D. Wang, and J. L. Hou, "Gas emission characteristics of tectonic coal and microscopic explanation of pore structure," Journal of China Coal Society, vol. 43, no. 12, pp. 3404-3410, 2018.

[8] Z. H. Wen, Y. P. Yang, Q. Wang, and B. H. Yao, "Mechanism and characteristics of $\mathrm{CH}_{4} / \mathrm{CO}_{2} / \mathrm{H}_{2} \mathrm{O}$ adsorption in lignite molecules," Geofluids, vol. 2021, Article ID 5535321, 11 pages, 2021.

[9] J. W. Yue, Z. F. Wang, J. S. Chen, M. H. Zheng, Q. Wang, and X. F. Lou, "Investigation of pore structure characteristics and adsorption characteristics of coals with different destruction types," Adsorption Science and Technology, vol. 37, no. 7-8, pp. 623-648, 2019.

[10] F. Du and K. Wang, "Unstable failure of gas-bearing coal-rock combination bodies: insights from physical experiments and numerical simulations," Process Safety and Environmental Protection, vol. 129, pp. 264-279, 2019.

[11] S. B. Chen, X. H. Xia, Y. Qin, C. Q. Fu, and L. Hu, "Classification of pore structures in shale gas reservoir at the Longmaxi Formation in the south of Sichuan Basin," Journal of China Coal Society, vol. 38, no. 5, pp. 760-765, 2013.

[12] Z. W. Liao, X. F. Liu, D. Z. Song et al., "Micro-structural damage to coal induced by liquid $\mathrm{CO}_{2}$ phase change fracturing," Natural Resources Research, vol. 30, no. 2, pp. 1613-1627, 2020.

[13] J. Zhu, B. Zhang, Y. Zhang, J. Tang, and Y. D. Jiang, "Coal pore characteristics in different coal mine dynamic disasters," Arabian Journal of Geosciences, vol. 11, no. 17, pp. 1-10, 2018.

[14] Y. B. Yao and D. M. Liu, "Petrophysical properties and fluids transportation in gas shale: a NMR relaxation spectrum analysis method," Journal of China Coal Society, vol. 43, no. 1, pp. 181-189, 2018.

[15] Q. Q. Gan, J. Xu, S. J. Peng, F. Z. Yan, R. F. Wang, and G. L. Cai, "Effects of heating temperature on pore structure evolution of briquette coals," Fuel, vol. 296, no. 2, article 120651, 2021.

[16] L. L. Si, J. P. Wei, Y. J. Xi et al., "The influence of long-time water intrusion on the mineral and pore structure of coal," Fuel, vol. 290, no. 1, article 119848, 2021.

[17] F. D. Xin, H. Xu, D. Z. Tang et al., "Pore structure evolution of low-rank coal in China," International Journal of Coal Geology, vol. 205, pp. 126-139, 2019.

[18] S. Tao, Z. J. Pan, S. D. Chen, and S. L. Tang, "Coal seam porosity and fracture heterogeneity of marcolithotypes in the Fanzhuang 
Block, southern Qinshui Basin, China," Journal of Natural Gas Science and Engineering, vol. 66, pp. 148-158, 2019.

[19] Z. P. Meng, S. S. Liu, B. Y. Wang, Y. D. Tian, and J. Wu, "Adsorption capacity and its pore structure of coals with different coal body structure," Journal of China Coal Society, vol. 40, no. 8, pp. 1865-1870, 2015.

[20] X. F. Liu, D. Z. Song, X. Q. He, Z. P. Wang, M. R. Zeng, and L. K. Wang, "Quantitative analysis of coal nanopore characteristics using atomic force microscopy," Powder Technology, vol. 346, pp. 332-340, 2019.

[21] J. P. Wei, S. H. Dai, Z. H. Wen, X. C. Ren, and M. G. Li, "Research on comprehensive characterization method of different rank coal porosity," Journal of Henan Polytechnic University (Natural Science), vol. 34, no. 3, pp. 305-310, 2015.

[22] Y. W. Ju, B. Jiang, Q. L. Hou, G. L. Wang, and A. M. Fang, "Structural evolution of nano-scale pores of tectonic coals in southern North China and its mechanism," Acta Geologica Sinica, vol. 79, no. 2, pp. 269-285, 2005.

[23] Y. Li, Y. G. Zhang, L. Zhang, and J. L. Hou, "Characterization on pore structure of tectonic coals based on the method of mercury intrusion, carbon dioxide adsorption and nitrogen adsorption," Journal of China Coal Society, vol. 44, no. 4, pp. 1188-1196, 2019.

[24] L. L. Qi, Z. F. Wang, H. M. Yang, and X. J. Chen, "Study on porosity of coal samples based on low temperature nitrogen adsorption method and mercury porosimetry," Coal Science and Technology, vol. 40, no. 8, pp. 36-39, 2012.

[25] L. Qin, S. G. Li, C. Zhai et al., "Joint analysis of pores in low, intermediate, and high rank coals using mercury intrusion, nitrogen adsorption, and nuclear magnetic resonance," Powder Technology, vol. 362, pp. 615-627, 2020.

[26] G. H. Ni, S. Li, S. Rahmin et al., "Effect of nitric acid on the pore structure and fractal characteristics of coal based on the low-temperature nitrogen adsorption method," Powder Technology, vol. 367, pp. 506-516, 2020.

[27] Z. Y. Wang, Y. P. Cheng, Y. X. Qi, R. P. Wang, L. Wang, and J. Y. Jiang, "Experimental study of pore structure and fractal characteristics of pulverized intact coal and tectonic coal by low temperature nitrogen adsorption," Powder Technology, vol. 350, pp. 15-25, 2019.

[28] J. T. Pu, Experimental Study of Pore Structure Characteristics of Full-Aperture Section of Middle and Low Rank Coal in Xinjiang Mining Area, [M. S. thesis], Xi'an University of Science and Technology, Xi'an, China, 2019.

[29] J. Shen, Y. Qin, and J. Zhao, "Maceral contribution to pore size distribution in anthracite in the South Qinshui Basin," Energy \& Fuels, vol. 33, no. 8, pp. 7234-7243, 2019.

[30] D. F. Zhao, Y. H. Guo, X. X. Mao, C. G. Lu, M. Li, and F. C. Qian, "Characteristics of macro-nanopores in anthracite coal based on mercury injection, nitrogen adsorption and FESEM," Journal of China Coal Society, vol. 42, no. 6, pp. 15171526, 2017.

[31] J. Zhu, M. Zhang, L. J. Chuan, J. Tang, and F. Zhao, "Experimental study on coal strain induced by methane sorption/desorption and effect of pore features," Chinese Journal of Rock Mechanics and Engineering, vol. 35, no. S1, pp. 2620-2626, 2016.

[32] C. X. Wang, W. Liu, and J. K. Liu, "Research on pore structure characteristics of gas coal in Fukang mining area," Industry and Mine Automation, vol. 45, no. 7, pp. 92-96, 2019.
[33] W. Q. Zhu, D. Z. Tang, H. Xu, Y. X. Yu, and L. Wang, "Characteristics of pore structure and specific surface area of lignite," Coal Geology \& Exploration, vol. 44, no. 6, pp. 59-63, 2016.

[34] L. Wang, G. X. Zhang, J. Liu, X. J. Chen, and Z. Q. Li, “Effect of the pore structure on adsorption and diffusion migration of different rank coal samples," Energy \& Fuels, vol. 34, no. 10, pp. 12486-12504, 2020.

[35] P. Chen and X. Y. Tang, "The research on the adsorption of nitrogen in low temperature and micro-pore properties in coal," Journal of China Coal Society, vol. 26, no. 5, pp. 552556, 2001. 